\title{
Cerebral Toxocara canis larval infection presenting as delayed encephalopathy and nonconvulsive status epilepticus: a case report
}

\author{
Jung-Ju Lee, Kyusik Kang, Woong-Woo Lee, Byung-Kun Kim, Jong-Moo Park, Ohyun Kwon, Soohyun Cho \\ Department of Neurology, Nowon Eulji Medical Center, Eulji University, Seoul, Korea
}

\begin{abstract}
Adult cerebral Toxocara canis larval infections are rare. Humans are infected by contacting soil that contains infectious eggs or by eating undercooked meat. We encountered a patient with cerebral T. canis infection who showed recurrent altered mentality with nonconvulsive status epilepticus (NCSE). A 71-year-old male was admitted with complaints of drowsy mentality and abnormal behavior. Magnetic resonance imaging revealed non-enhanced multiple gliosis and calcified lesions. Electroencephalography revealed NCSE, which was cured with valproate. However, he was readmitted because of recurrence of symptoms. On cerebrospinal fluid examination, T. canis antibodies were observed. Subsequently, he was treated with albendazole and corticosteroids. Although corticosteroid improved his symptoms, the therapy could not be continued because the patient developed septic shock. This is the first report of cerebral T. canis larval infection presenting as delayed encephalopathy and NCSE. In patients with unexplainable progressive encephalopathy, particularly when gliosis or calcified lesions are observed on neuroimaging, cerebral T. canis larval infection should be considered and treated promptly.
\end{abstract}

Keywords: Toxocara canis, Status epilepticus, Albendazole, Adrenal cortex hormones

\section{Introduction}

Toxocariasis is a zoonosis caused by the larvae of Toxocara canis. Dogs can transmit the parasite to humans or domestic animals via egg-contaminated soil. Humans are infected by ingestion of embryonated eggs, either by contacting soil containing infectious eggs or by eating undercooked meat of an intermediate host of the parasite [1-3]. Most human infections are subclinical or self-limited and are usually observed in patients at a young age $[2,4,5]$.

Herein, we report a case of cerebral T. canis larval infection (CTLI) in an elderly patient diagnosed based on recurrent mental changes, nonconvulsive status epilepticus (NCSE), and cerebrospinal fluid (CSF) analysis. Written informed consent was obtained for publication of this case report and accompanying images.

\section{Case Report}

A 71-year-old male with diabetes visited our emergency department with complaints of drowsy mentality and abnormal behavior. The symptoms developed insidiously and progressively worsened. One week before admission, the patient experienced difficulty in articulation caused by limitations of tongue movement. He then developed general weakness and somnolence. One day before admission, he forgot to depart at his bus stop. His family members stated that he was unaware of his location when he was speaking to them over the phone. His past medical history included diabetes, and his blood sugar level was relatively well controlled with oral hypoglycemic dugs for years. He had not suffered from hypoglycemia during treatment for diabetes.

On admission, he was drowsy and responded slowly to external stimuli. Intermittent motionless staring was noticed during ex-

Received: February 3, 2021 Revised: March 3, 2021 Accepted: March 10, 2021

Correspondence: Jung-Ju Lee

Department of Neurology, Nowon Eulji Medical Center, Eulji University, 68 Hangeulbiseok-ro, Nowon-gu, Seoul 01830, Korea

E-mail: sss331@eulji.ac.kr

ORCID: https://orcid.org/0000-0001-7498-5656

Copyright (C) 2021 by The Korean Encephalitis and Neuroinflammation Society

This is an open access article distributed under the terms of the Creative Commons Attribution Non-Commercial License (http://creativecommons.org/licenses/by-nc/4.0/) which permits unrestricted non-commercial use, distribution, and reproduction in any medium, provided the original work is properly cited. 
amination. Vital signs were stable. Routine blood tests revealed hyponatremia (sodium concentration, $129 \mathrm{mmol} / \mathrm{L}$; normal value, 136-145 mmol/L), hyperglycemia (glucose level, $297 \mathrm{mg} /$ $\mathrm{dL}$; normal value, $70-110 \mathrm{mg} / \mathrm{dL}$ ), and elevated serum creatinine level (2.0 mg/dL; normal value, 0.6-1.2 mg/dL). Common blood cell count and differential blood cell count revealed no abnormalities. Eosinophil count was within the normal range (4.6\%; normal value, $0 \%-7 \%$ ). Brain magnetic resonance imaging (MRI) revealed periventricular extensive leukoaraiosis and moderate cortical atrophy. Low-signal ovoid lesions were observed within the leukoaraiosis on the fluid-attenuated inversion recovery image, and multiple, low-signal, round lesions were observed on the gradient-echo image, consistent with gliosis and calcification, respectively (Figure 1). No enhanced lesions were observed on the gadolinium-enhanced T1 image. Initial electroencephalography (EEG) showed frontal intermittent delta activity with a normal background alpha rhythm (Figure 2A). The patient was tentatively diagnosed with metabolic encephalopathy due to malnutrition and dehydration. However, administrations of intravenous normal saline and thiamine failed to improve the patient's mentality, despite correct- ing the previously detected abnormalities in the laboratory tests. A few days later, the patient's drowsiness progressed, and he showed semipurposeful and fumbling hand movement with decreased responsiveness. Follow-up EEG showed recurrent asymmetric rhythmic delta activities with fluctuating frequency and amplitude (Figure 2B). Valproic acid was administered intravenously at a loading dose of 1,000 mg and maintenance dose of $300 \mathrm{mg}$ three times a day. The patient gradually improved to a nearly premorbid mental state and was discharged on hospitalization day 17 .

Two months after discharge, however, he was readmitted to the hospital because of aggravated mentation. He responded slowly to verbal commands, and he had become somnolent 5 days before readmission. Brain computed tomography and diffusion-weighted MRI did not demonstrate any acute lesion. Follow-up EEG revealed persistent seizure activity (Figure 3A, B) that resolved with intravenous lorazepam, which was accompanied by transient clinical improvement. Intravenous fosphenytoin (loading dose, $30 \mathrm{mg} / \mathrm{kg}$; maintenance dose, $450 \mathrm{mg} /$ day) was administered but failed to restore his mentality. Levetiracetam (1,000 mg twice per day) and pregabalin (150 mg twice per

Figure 1 Brain magnetic resonance image of a patient with cerebral Toxocara canis larval infection
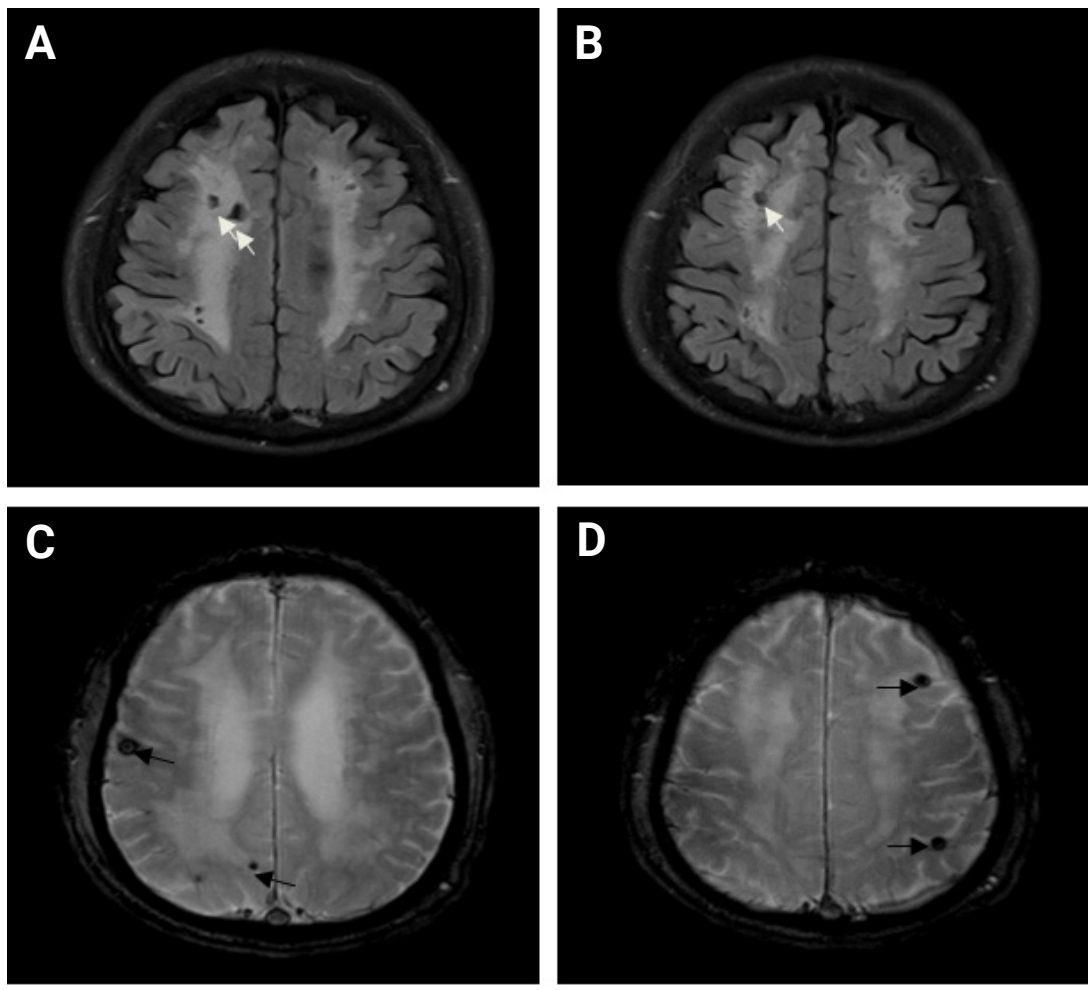

(A, B) Multiple low-signal lesions in periventricular leukoaraiosis are observed on the fluid-attenuated inversion recovery image (white arrows). (C, D) Multiple calcified lesions are observed on the gradient-echo image (black arrows). 
Figure 2 Electroencephalography performed at the first admission

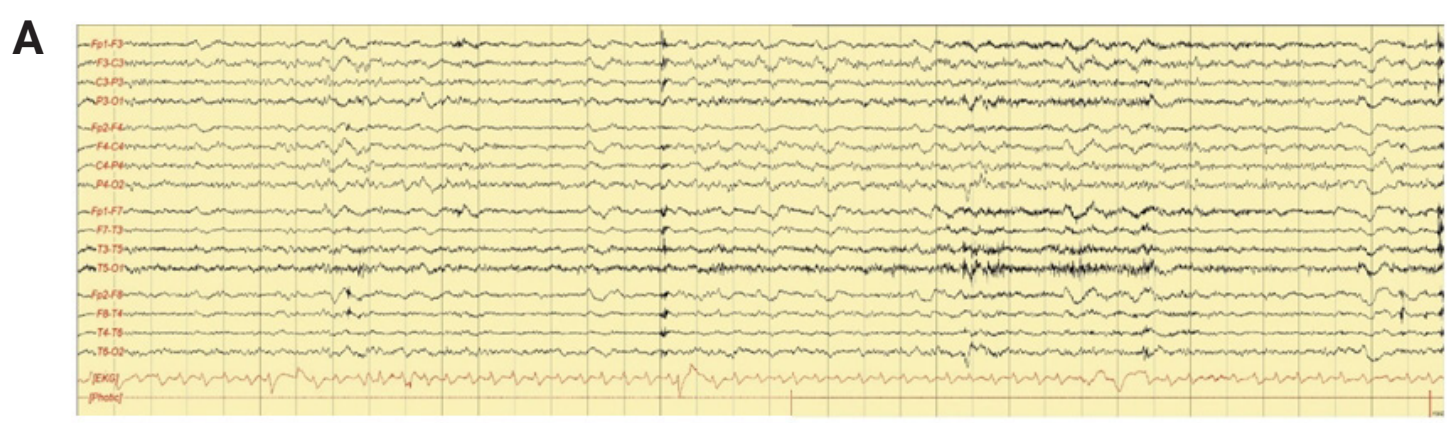

B

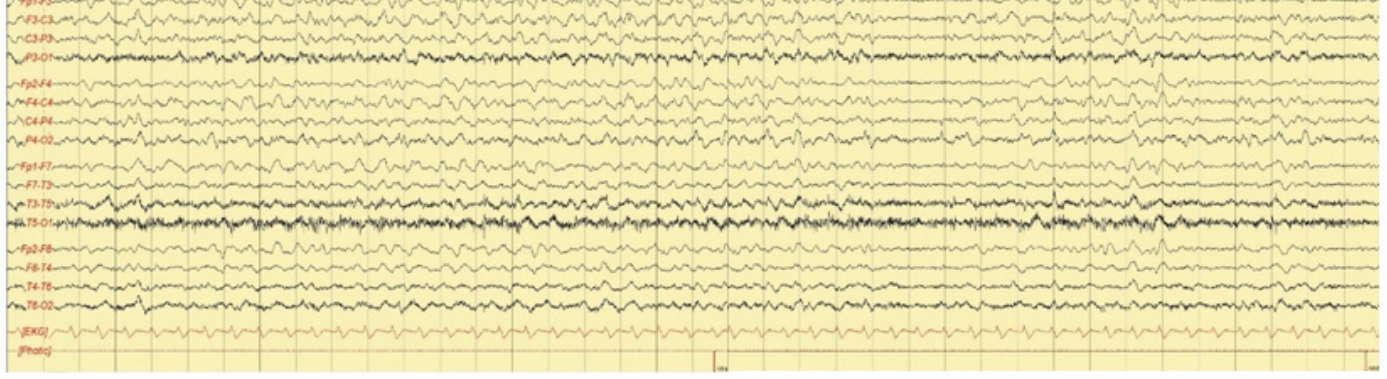

(A) Frontal-dominant intermittent rhythmic delta activities are observed. (B) Intermittent, asymmetric, and rhythmic delta activities are observed with fluctuating frequency and amplitude, which ceased with a low-voltage alpha rhythm.

Figure 3 Electroencephalography performed at the second admission

A

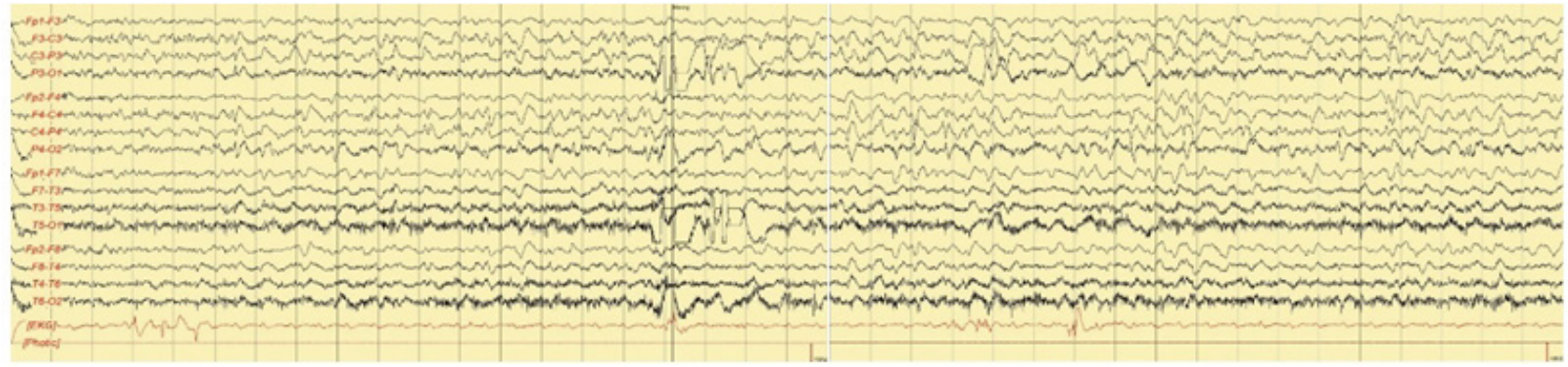

B

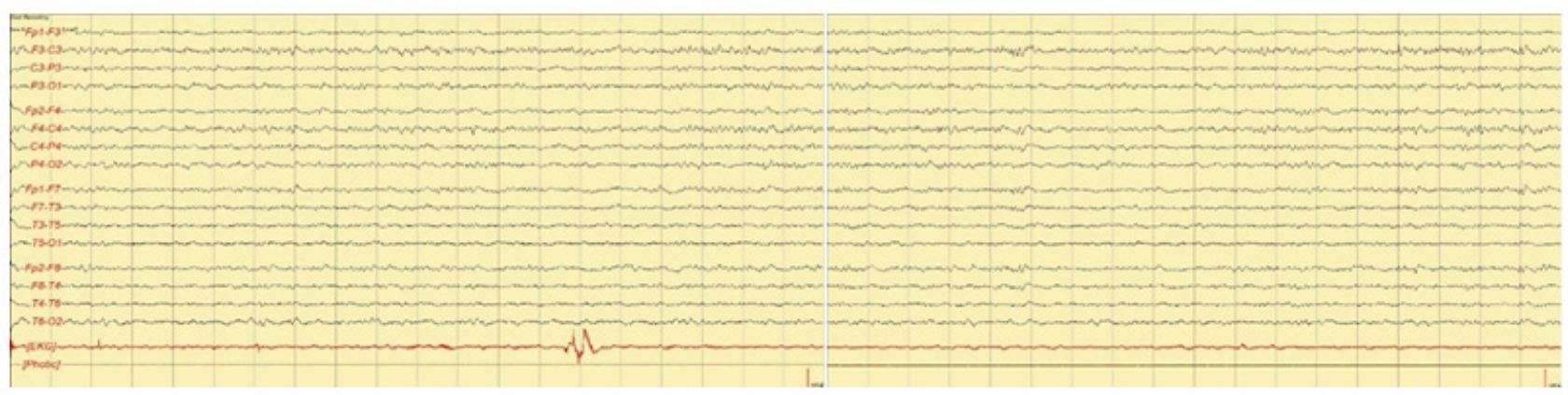

(A) Frontal fast discharges and asymmetric quasi-rhythmic delta activities are observed alternately with a fluctuating amplitude. (B) After intravenous injection of lorazepam, these activities disappeared with clinical improvement. 
day) were added, and the patient improved partially; he became alert and could make eye contact with other people. Valproate was discontinued because serum ammonia level was elevated on hospitalization day 5 . To exclude other encephalopathic disorders, CSF examination was performed and revealed no pleocytosis or protein elevation (white blood cell count, 0/L; CSF protein level, $24.7 \mathrm{mg} / \mathrm{dL}$ ). A parasite-specific immunoglobulin (Ig) G antibody test performed using enzyme-linked immunosorbent assay (ELISA) revealed an IgG of T. canis that exceeded the normal limit to an absorbance of 0.352 and 0.276 in the serum and CSF, respectively (normal absorbance value, <0.250). Other tests, including CSF 14-3-3 protein, CSF aquaporin-4 antibody, serum and CSF paraneoplastic antibodies (anti-Hu, -Yo, -Ma2, -CRMP5, -amphiphysin, and -Ri), serum and CSF antithyroid autoantibodies (thyroglobulin and thyroid peroxidase antibodies), and serum and CSF antibodies targeting neuronal cell surface antigens and ligand-gated ion channels were negative. The patient's family reported that he often consumed raw meat and liver. Stool examination revealed no helminth egg. Computed tomography and ultrasonography of the chest, abdomen, and pelvis revealed no specific lesion.

Treatment with oral albendazole (400 mg twice per day), started on hospitalization day 25 and maintained for 2 weeks, failed to show additional improvement in the patient's mentality. Intravenous corticosteroid (methylprednisolone, 1,000 mg/day) was started on hospitalization day 34 and maintained for 5 days, after which he was administered oral prednisolone (60 mg/day). Significant improvement in mentality, including an increase in spontaneous verbal output and persistent eye contact, was observed after 1 week of corticosteroid treatment. In addition, the seizure activity on EEG disappeared, except for interictal epileptiform discharges (Figure 4). However, corticosteroid therapy had to be discontinued because the patient developed septic shock from an aggravated sacral sore. Corticosteroid therapy was replaced with intravenous Ig (400 mg/ $\mathrm{kg}$ for 5 days), which failed to restore his mentality. He was transferred to a nursing hospital while maintaining antiepileptic drugs and a small daily dose of prednisolone (10 mg/day). He slowly progressed to a vegetative state and expired 3 years after discharge from our hospital.

\section{Discussion}

Toxocariasis, also called visceral larva migrans, is characterized by paratenic larvae that migrate through organs. Depending on the involved organ, it can present with symptoms similar to rheumatism, asthma, or neurologic disorders. Central nervous system involvement of $T$. canis larvae is rare, and clinical manifestation is rarer. Most patients with autopsy-proven infection presented no clinical symptoms during their lifetimes [4,6]. The mechanisms involved in CTLI are not fully understood yet.

Our patient showed no active lesions on brain imaging; only gliosis and calcified lesions without enhancement were observed. CSF examination revealed no pleocytosis or protein elevation. Treatment with albendazole had little effect on the patient's clinical course, but corticosteroids had a significant effect. Therefore, we assumed that the larvae had been inactivated before treatment. The encephalopathy and seizure activity in our patient might be associated with delayed inflammatory reactions. A previous study reported that clinical and radiological improvement in some patients could be achieved with steroid therapy without antihelminthic drugs [6]. Autoimmune inflammatory mechanisms might have played an important role in the progressive encephalopathy and NCSE observed in our patient.

Figure 4 Electroencephalography after steroid therapy

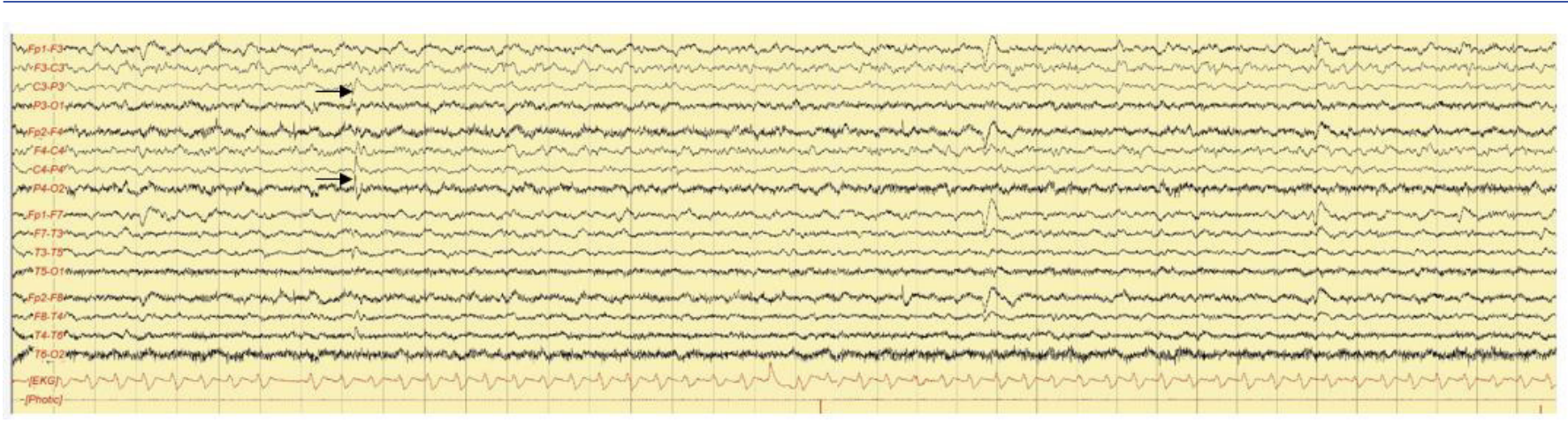

Seizure activities disappeared, except for occasional interictal epileptiform discharges (arrows). The background alpha rhythm was relatively well regulated. 
Cytokines produced against the parasite can compromise vulnerable neurons, resulting in autoimmune encephalopathy [5]. Furthermore, prolonged and elevated levels of cytokines can induce neuronal damage. Microglia-driven neuroinflammation produces neurotoxic products, such as nitric oxide, reactive oxygen and proinflammatory cytokines, which cause inflammation and neurodegeneration [7]. Corticosteroid can be helpful for autoimmune vasculitis, eosinophilic meningitis, and prevention of progression of neurodegeneration in patients with CTLI $[5,8]$.

The diagnosis of CTLI is challenging. Although eating raw beef is prevalent in Korea, the diagnosis might be missed without meticulous history taking. Neuroimaging and laboratory findings can be nonspecific and mimic those of various neurological disorders, such as stroke, epilepsy, and neurodegenerative disorders $[5,9,10]$. Only after clinical suspicion can the correct diagnosis be obtained.

To the best of our knowledge, this is the first report of CTLI presenting as delayed encephalopathy and NCSE. For patients with unexplainable progressive encephalopathy and NCSE, particularly when gliosis or calcified lesions are observed on neuroimaging, CTLI should be considered, and a CSF ELISA test for parasitosis should be performed. The diagnosis was delayed in our patient, which might have resulted in the poor outcome. Timely diagnosis with prompt treatment can prevent significant neurological damage and mortality.

\section{Conflicts of Interest}

No potential conflict of interest relevant to this article was reported.

\section{Author Contributions}

Conceptualization: JJ Lee, JM Park; Data curation: K Kang, WW Lee, JM Park; Formal analysis: JM Park; Investigation: K Kang, O Kwon; Methodology: JM Park, S Cho; Visualization: BK Kim, S Cho; Resources: K Kang; Software: K Kang, WW Lee, O Kwon, S Cho; Supervision: JJ Lee, BK Kim; Validation: JJ Lee, BK Kim, O Kwon; Writing-original draft: JJ Lee; Writing-review and editing: JJ Lee.

\section{References}

1. Sommer C, Ringelstein EB, Biniek R, Glöckner WM. Adult Toxocara canis encephalitis. J Neurol Neurosurg Psychiatry 1994;57:229-231.

2. Choi D, Lim JH, Choi DC, et al. Transmission of Toxocara canis via ingestion of raw cow liver: a cross-sectional study in healthy adults. Korean J Parasitol 2012;50:23-27.

3. Magnaval JF, Glickman LT, Dorchies P, Morassin B. Highlights of human toxocariasis. Korean J Parasitol 2001;39:1-11.

4. Magnaval JF, Galindo V, Glickman LT, Clanet M. Human Toxocara infection of the central nervous system and neurological disorders: a case-control study. Parasitology 1997;115:537-543.

5. Fan CK, Holland CV, Loxton K, Barghouth U. Cerebral toxocariasis: silent progression to neurodegenerative disorders? Clin Microbiol Rev 2015;28:663-686.

6. Sánchez SS, García HH, Nicoletti A. Clinical and magnetic resonance imaging findings of neurotoxocariasis. Front Neurol 2018;9:53.

7. Tufekci KU, Genc S, Genc K. The endotoxin-induced neuroinflammation model of Parkinson's disease. Parkinsons Dis 2011;2011:487450.

8. Chotmongkol V, Sawanyawisuth K, Thavornpitak Y. Corticosteroid treatment of eosinophilic meningitis. Clin Infect Dis 2000;31:660-662.

9. Çelik T, Kaplan Y, Ataş E, Öztuna D, Berilgen S. Toxocara seroprevalence in patients with idiopathic Parkinson's disease: chance association or coincidence? Biomed Res Int 2013;2013:685196

10. Richartz E, Buchkremer G. Cerebral toxocariasis: a rare cause of cognitive disorders. A contribution to differential dementia diagnosis. Nervenarzt 2002;73:458-462. 